\title{
FUSION OF LOCATION FINGERPRINTING AND TRILATERATION BASED ON THE EXAMPLE OF DIFFERENTIAL WI-FI POSITIONING
}

\author{
G. Retscher \\ Dept. of Geodesy and Geoinformation, TU Wien - Vienna University of Technology, Gusshausstrasse 27-29, 1040 Vienna, Austria \\ guenther.retscher@tuwien.ac.at
}

KEY WORDS: Indoor positioning, Wi-Fi, RSS, Location fingerprinting, Trilateration, Differential positioning, Dynamic radio maps, Fusion

\begin{abstract}
:
Positioning of mobile users in indoor environments with Wireless Fidelity (Wi-Fi) has become very popular whereby location fingerprinting and trilateration are the most commonly employed methods. In both the received signal strength (RSS) of the surrounding access points (APs) are scanned and used to estimate the user's position. Within the scope of this study the advantageous qualities of both methods are identified and selected to benefit their combination. By a fusion of these technologies a higher performance for Wi-Fi positioning is achievable. For that purpose, a novel approach based on the well-known Differential GPS (DGPS) principle of operation is developed and applied. This approach for user localization and tracking is termed Differential Wi-Fi (DWi-Fi) by analogy with DGPS. From reference stations deployed in the area of interest differential measurement corrections are derived and applied at the mobile user side. Hence, range or coordinate corrections can be estimated from a network of reference station observations as it is done in common CORS GNSS networks. A low-cost realization with Raspberry Pi units is employed for these reference stations. These units serve at the same time as APs broadcasting Wi-Fi signals as well as reference stations scanning the receivable Wi-Fi signals of the surrounding APs. As the RSS measurements are carried out continuously at the reference stations dynamically changing maps of RSS distributions, so-called radio maps, are derived. Similar as in location fingerprinting this radio maps represent the RSS fingerprints at certain locations. From the areal modelling of the correction parameters in combination with the dynamically updated radio maps the location of the user can be estimated in real-time. The novel approach is presented and its performance demonstrated in this paper.
\end{abstract}

\section{INTRODUCTION}

Wi-Fi is one of the most widely used signal-of-opportunity ( $\mathrm{Li}$ and Rizos, 2012) which can be employed for positioning and tracking of mobile users in indoor environments if the required infrastructure is available (Mautz, 2012; Retscher, 2016). It is a flexible data communication protocol implemented to extend or substitute for a wired local area network, such as Ethernet. The bandwidth of $802.11 \mathrm{~b}$ is 11 Mbits and it operates at $2.4 \mathrm{GHz}$ frequency (Wang et al., 2006). It is originally a technology for short-range wireless data communication and is typically deployed as an ad-hoc network in a hot-spot fashion in the areas where a wireless Internet access is needed. IEEE 802.11 is currently the most utilized Wi-Fi technology. Wireless networks are built by attaching a device called access point (AP) to the edge of a wired network. In the infrastructure topology, APs are the central control point which forward traffic between terminals of the same cell and bridges traffic to wired LAN. Clients communicate with the AP using a wireless network adapter that is similar to a traditional Ethernet adapter. Beacon frames are transmitted in IEEE 802.11 Wi-Fi for network identification, broadcasting network capabilities, synchronization and for other control and management purposes. Thus, APs transmit beacons periodically according to the beacon interval parameter in this topology. A synchronization function is defined that keeps timers of all terminals synchronized to the AP clock by using the timestamp information of the beacon frames. The IEEE 802.11 MAC (Media Access Control) protocol utilizes carrier sensing based contention. The carrier sensing is based on energy detection or signal quality. The standard specifies the RSS that measures RF (radio frequency) energy by the radio. RSS of up to 8 bits (256 levels) are supported, but the absolute accuracy is not specified (Kotanen et al., 2003). The transmission power is the major factor that has direct influence on the effective range. Hence, the RSSs and MAC addresses of the APs are locationdependent information that can be adopted for positioning purpose. An observable associated with a MAC address of an AP consist of the following information: (1) the unique MAC address of the RF transmitter, (2) the location of the RF transmitter, and (3) the effective range of the signal, or the size of the signal coverage area of the RF transmitter (Chen et al., 2012). For localization of a mobile device either cell-based solutions or trilateration and location fingerprinting are commonly employed (Retscher, 2016). A fusion of localization technologies is proposed and investigated for the example of Wi-Fi positioning. A higher performance is expected to be achieved using a meaningful and careful selection of the advantages of location fingerprinting and trilateration. The potential of this strategy is demonstrated in this paper. Within the scope of this study, the advantageous qualities of both methods are identified and selected to benefit their combination.

The paper is organized as follows: In section 2 the properties of the two methods are identified and assessed followed by an introduction of the DWi-Fi positioning approach and its evaluation in section 3. Section 4 shows examples for the deduction of radio maps and section 5 discusses the concept of fusion of location fingerprinting and trilateration. Finally, brief conclusions are given. 


\section{IDENTIFICATION AND ASSESSMENT OF THE PROPERTIES OF LOCATION FINGERPRINTING AND TRILATERATION USING WI-FI}

Apart from cell-based positioning solutions mainly location fingerprinting and trilateration are applied for localization of a mobile user with Wi-Fi. The main disadvantage in Wi-Fi positioning with these two methods, however, is that its performance is significantly affected by the fluctuation and various propagation effects on the scanned Wi-Fi RSS values. Temporal and spatial variations as well as high signal noise caused by the surrounding environment and its changes lead usually to low achievable positioning accuracies on the several meter level (Stojanović and Stojanović, 2014). In the following the basics of both methods are briefly reviewed and their advantages and drawbacks identified.

\subsection{Location Fingerprinting}

RF-based fingerprinting refers to the type of algorithms that first collect features, i.e., so-called fingerprints, of RSS distributions and then estimates the user's location by matching online measurements with the closest predetermined location fingerprints included in a so-called fingerprinting or radio map. Figure 8 shows an example for a radio map in the field test site. Several location fingerprinting-based positioning algorithms have been developed, such as pattern recognition (Liu et al., 2007), which can be classified as deterministic or probabilistic. Deterministic location estimation is based on the similarity of the RSS measurements and the fingerprints in the database. Each RSS sample is not used separately, but the sample averages of different transmitters are collected into a vector and used to estimate the mobile device's location (Bahl and Padmanabhan, 2000). The simplest ways to determine the location is the $k$-nearest neighbour or the $k$-weighted nearest neighbour algorithm where $k$ reference points in the radio map are compared to the observed measurements to select $k$ reference points with the nearest RSS values. The Euclidean norm is usually used as a vector distance measure (Honkavirta, 2008). The probabilistic approach (Roos et al. 2002) exploits the sample of measurements collected during the training phase more efficiently than the deterministic methods. The idea behind is to compute the conditional probability density function (PDF) of the unknown position. The prior distribution is usually considered to be uniform. Using Bayes' theorem (Koch, 2000) and the measurements the posterior PDF can be calculated. The fingerprints hold information about the signal characteristics across the cells. The normalized histogram pattern measured at the reference point from a transmitter is interpreted as the distribution of the RSS sample from the transmitter. Several approaches for the calculation are available, e.g. the histogram method and the kernel method (see Honkavirta, 2008; Roos et al. 2002). A particle filter (Gordon et al., 1993), for instance, can be used for the estimation of most likely location of the user.

\subsection{Trilateration}

Trilateration is a conventional algorithm used in surveying, as well as in the RSS-based techniques. It is used to determine the position of the intersection of at least three spherical surfaces given the centres and radii of those spheres (Retscher, 2016). In RSS-based approaches trilateration is based on the nature of the RSS which varies with the changes of distance between transmitters and receivers. Theoretically, the RSS decreases with the transmitted energy propagating into space. A number of models, termed path loss models, have been developed to establish the relationship between the RSS and propagating distances. The ranges between the transmitters and the receiver can be estimated by inverting the model using the measured RSS of the signals (Retscher et al., 2012). In most environments, a logarithmic function can be applied to obtain the relationship between the measured RSS and the range to the transmitter, i.e., the AP in case of $\mathrm{Wi}-\mathrm{Fi}$ positioning. An example for such a logarithmic relationship is shown in Figure 4. A typical logarithmic path loss model is the so-called one-slope model (Retscher and Tatschl, 2016). It is a very simple empiric model which is based on the principle of the free space loss of the signals. The damping depends then only on the logarithmic distance between the transmitter and receiver and the reference RSS in the form:

$$
P(d)=P_{0}+10 \gamma * \log _{10}(d)
$$

where $P$ is the received empirical RSS, $P_{0}$ the reference RSS in $1 \mathrm{~m}$ distance, $\gamma$ the damping factor and $d$ the distance between the transmitter and receiver. The damping factor $\gamma$ can vary significantly which can then cause large errors for the RSS to distance conversions. In free space, $\gamma$ equals 2 , which aligns the log-distance path loss model well with the free space propagation model. In outdoor and indoor non-line-of sight (NLOS) areas, $\gamma$ increases and its normal range is between 2 to 6. In indoor line-of sight (LOS) conditions, especially in corridors, this value can fall to less than 2 . In the performed experiments described in section 3 the coefficients $P_{0}$ and $\gamma$ are estimated using a least-squares adjustment from the RSS measurements of the different smartphones.

\subsection{Disadvantages and Drawbacks}

As described above the IEEE $802.11 \mathrm{~b}$ standard uses RF signals in the $2.4 \mathrm{GHz}$ band, which is attractive because it is licensefree, however, it does suffer from inherent disadvantages. In the $2.4 \mathrm{GHz}$ band, microwave ovens, Bluetooth devices, cordless phones and other devices are sources of interference. Moreover, signal propagation suffers from server multipath fading effects due to reflection, refraction, diffraction and absorption by structures and humans. As a result, a transmitted signal can reach a receiver through different paths, each having its own amplitude and phase. These different components are captured by the receiver and a distorted version of the transmitted signal is reconstructed. Furthermore, changes in the environmental conditions such as temperature and humidity affect the strength of the received signals to a large extent. Consequently, the RSS values received by a Wi-Fi card at a fixed location vary with time and physical conditions of the surrounding environment (Chang et al., 2010). Furthermore, the presence of people and the user himself affects the scanned RSS values significantly. Signals of APs may be blocked or the RSS is lowered due to the body of the person to be localized. From the tests of $\mathrm{Hu}$ (2013) could be seen that typically Wi-Fi signals are affected when people are located physically between the APs and the mobile device. The main reason for this is that $2.4 \mathrm{GHz}$ signals can be greatly attenuated by water and the human body consist of about $70 \%$ water

The limitation of Wi-Fi trilateration based on RSS measurements is that most of the theoretical path loss models are subject to free space propagation (i.e., LOS) or signal propagation in a simple environment with a limited number of reflectors and obstacles. For example, in an office building, metal window frames and pipes passing through rooms can be reflectors of Wi-Fi radio frequency signals. In real terms, the environment can be tremendously complex and therefore, 
difficult to deal with through the assumptions or conditions listed in the physical or theoretical models. These detrimental effects will degrade the accuracy of the distance estimated using the inversed path loss models (Retscher et al., 2012).

In general, location fingerprinting is more robust to environmental effects on the RSS than using the RSS-based trilateration algorithm. This is because the location fingerprinting algorithm constructs a search space according to either a simulated environmental model (e.g., a model of the building) or previously-measured RSS distributions in the radio maps using a site survey. As mentioned above this process is done in the training phase. If using only simulated physical environmental models where the distribution of the RSS is simulated by models, such as ray tracing, according to the given environmental conditions, the characteristics of every object in the building have to be defined. The major problem is that physical models cannot accurately represent the reality which leads to inaccurate RSS distributions and consequently degrades the positioning accuracy. Higher positioning accuracies can be achieved when a site survey is conducted in the training phase and with the measured RSS values the database of fingerprints is established. The advantage of constructing the database in that sense is that it can be used to consider a great number of detrimental effects from the surrounding environment, such as reflections and obstructions, into the radio maps and thus increases the accuracy for finding the best matching position based on RSS in the positioning phase (Retscher et al., 2012). The main drawback of fingerprinting is the very labour consuming workload in the training phase for establishing the fingerprinting database and their graphical representation in radio maps. Furthermore, heterogeneous mobile devices measure RSS differently. Spatial interpolation techniques are usually employed for densification of the database and the respective radio map and it is advisable that different mobile devices are used for RSS measurements in the training phase to form a joint database. Because of these disadvantages a new approach has been proposed by the author where continuous RSS scans at reference stations are conducted.

\subsection{Problem Solution Approach}



Figure 1. Raspberry Pi unit including an internal Wi-Fi card and an external USB Wi-Fi adapter with rod areal antenna

The aim of this study is to achieve a performance improvement and reduction of the labour intensive RSS site survey in the training phase for indoor Wi-Fi localization and tracking using location fingerprinting and (tri)lateration. Thereby the recorded RSS values at the user's device are corrected and improved using continuous RSS scans at reference stations deployed in the area of interest (see Figure 2). For a low-cost realization Raspberry Pi units serve as reference stations and APs at the same time scanning and emitting Wi-Fi signals. A Raspberry Pi is a low-cost credit card sized computer (see Figure 1). An USB Wi-Fi adapter with a rod areal antenna is plugged into each Raspberry Pi and the internal Wi-Fi card is used in addition. Using these devices the RSS scans are recorded together with the MAC addresses of the Wi-Fi APs continuously.

\section{ASSESSMENT OF THE DIFFERENTIAL WI-FI APPROACH AND ITS EVALUATION}

In this section, the novel differential approach based on the well-known DGPS operational principle and experiments conducted in a multi-storey building are presented.

\subsection{DWi-Fi Operational Concept}

As wireless communication channels in indoor environments are generally noisy which makes the measured RSS a complex function of distance and environmental factors. Temporal and spatial fluctuation and signal noise of the recorded RSS reduces the accuracy of the location estimation considerably; especially indoors where NLOS propagation and attenuation caused by walls, other structures, and even people cause significant fluctuations of the RSS measurements. To overcome this drawbacks a novel approach termed differential Wi-Fi (DWiFi) by analogy to DGPS is developed. In this approach, the RSS to distance relationship is improved with range corrections which are applied to the deducted ranges to the APs. Thus, the inherent detrimental effects in Wi-Fi trilateration are minimized. Raspberry Pi units measure continuously the RSS to all other 'visible' APs similar as it is done on the mobile user's side. The RSS and the deduced differential corrections are obtained from a comparison with the known ranges between the AP reference stations. Figure 2 illustrates the DWi-Fi approach with a network of Raspberry Pi units. As an example three reference stations RS 1 to 3 are deployed at known locations. Then it is possible to derive areal correction parameters (i.e., so-called Flächenkorrekturparameter FKP) in the reference AP network similar as it is done in RTK-GNSS positioning in a continuous operating reference station (CORS) network. The FKP are then applied to the current RSS scans from the mobile user. Apart from the Raspberrry Pi units which serve at the same time as reference stations and APs the mobile user can record the RSS of all 'visible' $n-1$ APs from available Wi-Fi networks in the surrounding as depicted in Figure 2.

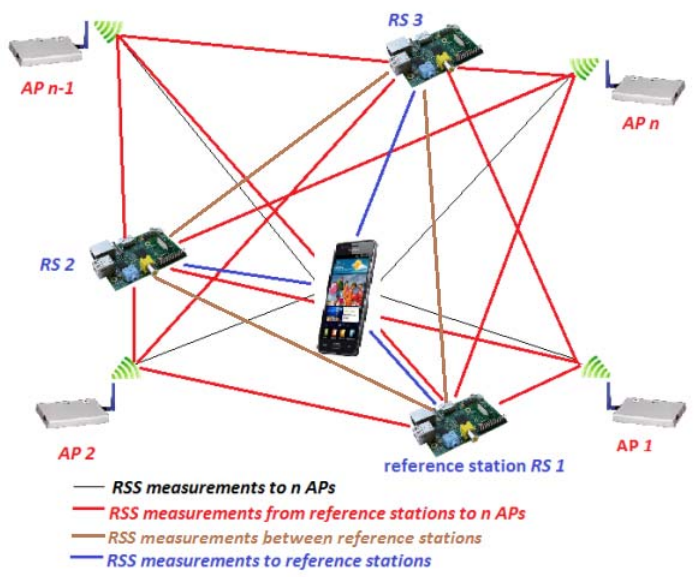

Figure 2. Network of AP and reference stations for DWi-Fi

A simple mathematical formulation describes the calculation of the correction parameters. Namely, the current RSS scans are compared with the nominal RSS obtained from the known RS position. Then the theoretical RSS $\left(R S S_{\text {calc }}\right)$ can be derived from the relationship between the range to the APs and the currently measured RSS $\left(R S S_{o b s}\right)$. Simple differentiation yields the RSS corrections $\left(R S S_{\text {corr }}\right)$ for the current time measurement epoch $t$ as described in equation (2):

$$
R S S_{\text {corr }}(t)=R S S_{\text {calc }}-R S S_{\text {obs }}(t)
$$


The user applies these corrections to improve his RSS scans and to reduce errors dependent on the transmitter and caused by various propagation phenomena. Thereby the synchrony between RS and the smartphone is a necessary requirement, because the correction values contain temporal variations. It is evident that the calculated corrections fulfil their validity in close surrounding to the RS because the damping effects are dependent on the local conditions. Recall, this is also a requirement for DGPS positioning. A spatial interpolation becomes possible if several RSs are used. Then a 2D linear interpolation is applied to generate the FKP directly at the position of the user. The general plane equation can be formulated as:

$$
z(x, y)=a_{0}+a_{1} * x+a_{2} * y
$$

and can be derived with the three correction values of every RS. In matrix notation this leads to:

$$
\left[\begin{array}{l}
R S S_{\text {corr } 1} \\
R S S_{\text {corr } 2} \\
R S S_{\text {corr } 3}
\end{array}\right]=\left[\begin{array}{lll}
1 & x_{1} & y_{1} \\
1 & x_{2} & y_{2} \\
1 & x_{3} & y_{3}
\end{array}\right] *\left[\begin{array}{l}
a_{0} \\
a_{1} \\
a_{2}
\end{array}\right]
$$

The area correction parameters result unambiguously in:

$$
a=A^{-1} * Z
$$

With these three parameters the plane is defined precisely. The correction value at the user's position can be determined directly with the FKPs in equation (3). Nevertheless, from equation (3) is evident that the coordinates of the user must already be known before the interpolation. Thus, an iterative process has to be employed to determine the user's position starting with the user's position which has not been corrected so far. If more than two RSS observations for 2D trilateration are measured a redundancy is provided. The least-squares adjustment which is based on the minimization of the $\mathrm{L}_{2}$ norm enables the optimum estimation of the users' location. In the 2D case the following observation equation can be formulated:

$$
d_{U, A P_{i}}^{2}=\left(X_{U}-X_{A P_{i}}\right)^{2}+\left(Y_{U}-Y_{A P_{i}}\right)^{2}
$$

where $d_{U, A P_{i}}$ is the range between the user and $A P_{i}, X_{U}$ and $Y_{U}$ are the coordinates of the smartphone user and $X_{A P_{i}}$ and $Y_{A P_{i}}$ are the coordinates of the $A P_{i}$.

As start point for the calculation of the user location the wellknown Gauss-Markov model is used. As the function described in equation (6) is nonlinear, approximate locations for the user position are required for the estimation. For this calculation a particular weighting of the observations was applied. For an iterative compensation of linearization errors in the adjustment, a weighting iteration is applied following Schuh in the form:

$$
\begin{array}{ll}
p_{i}=\exp ^{-\left|\frac{v_{i}}{\sigma}\right|^{4.4^{0.05}}} & \text { for the } 1^{\text {st }} \text { and } 2^{\text {nd }} \text { iteration, } \\
p_{i}=\exp ^{-\left|\frac{v_{i}}{\sigma}\right|^{3.0^{0.05}}} & \text { for the following iterations, }
\end{array}
$$

with the weights $p_{i}$, the residuals $v_{i}$ and the variance of the unit weight $\sigma$. The respective parameters and unknown values including the position coordinates of the user are improved with every iteration. The iteration is carried out as long as the norm of the respected parameter vector falls below the limit value of $1 \mathrm{~cm}$.

A second approach based on the VLBI (Very Long Baseline Interferometry) operational principle was additionally developed. In contrast to VLBI, the difference calculation is not performed on the basis of propagation times, but on the measured RSS of the user and at the reference station. The range between the user and the AP is determined from the known distance between the RS and the AP with the range difference between the user to the AP. This range difference is derived from the RSS differences. Further information concerning this approach can be found in Retscher and Tatschl (2016). In principle, the range can be subtracted between the $\mathrm{AP}$ and the user with arbitrarily many RSs which leads to inherent further observations for the position determination of the user. Tests have shown that this approach does provide very similar performance in terms of achievable positioning accuracies than the one based on DGPS. Thus, in the following only results of the first DWi-Fi approach are presented.

\subsection{Differences of the DWi-Fi Concept to Existing Solutions}

The Chinese patent CN 103281777 A: 'Differential Positioning Method based on WiFi (Wireless Fidelity)' announced in September 2013 and claimes that a significant increase in positioning accuracies is achievable with location fingerprinting. The newly developed DWi-Fi localization approach presented in this study is different to the concept in the patent in several aspects. First of all it is based on trilateration and not fingerprinting. As already mentioned in section 2.3 fingerprinting requires a labour intensive workload for establishing a database of RSS fingerprints in the area of interest. To achieve suitable positioning accuracies at least on the room level resolution reference points must be located very dense. This is not required for trilateration. Furthermore, in the new approach the differencing is done on the RSS level of the RSS measurements of the Raspberry Pi units serving as reference stations as well as APs at the same time and the mobile devices of the users. This realizes a very low-cost solution. Due to a network of reference stations a more robust positioning solution with higher accuracy and integrity is achieved. The Chinese patent also claims that the approach is based on DGPS. A network-based solution, however, has not been patented. Also no server/client solution has been chosen in the developed concept as the users' privacy cannot be guaranteed if the calculation of the position of the user is performed on a server. Hence, it would be possible to track the user which is not foreseen here due to data protection rights.

In the paper of Chang et al. (2010) another differential approach for Wi-Fi positioning has been proposed. This approach differentiate between the novel approach developed in this study in similar aspects as the Chinese patent. Chang et al. (2010) also use location fingerprinting as preferred positioning method. Thus, the same advantages of our approach are applicable as stated above. Using the novel approach presented here a radio map generation is also possible without labour intensive training measurements. Chang et al. (2010) employ a single pair of APs instead of a network of reference stations. The authors believe that only one dual AP is not sufficient for large areas of interest. Such an approach provides only similar results with acceptable positioning accuracies in close proximity to the dual APs.

\subsection{Test Site for the DWi-Fi Concept Evaluation}

The DWi-Fi approach was tested for the first time on the ground floor of a multi-storey office building (see Figure 3). In the selected area three reference stations RS 1 to 3 have been deployed. 93 reference points distributed in a regular grid with distances of $2.5 \mathrm{~m}$ between them covering mainly three different areas, i.e., a foyer, a class room and an area with 
desktop computers. Thus, different signal propagation conditions regarding damping and shielding of the Wi-Fi signals are present. The ceiling height is approximately $5 \mathrm{~m}$ in the foyer as well as in the class room whereby in the entrance hall an area with an entresol exists. During the tests always six APs (CDEG-1 to -6) were visible. The blue line in the map indicates a kinematic positioning trajectory. The three Raspberry Pi's were located at the three APs CDEG-1, -3 and 6 (i.e., APs with RS in Figure 3). A disadvantage of the setup was that the location of the predeployed APs could not be selected under consideration of a good geometry for performing trilateration. They are located to provide a continuous Wi-Fi coverage throughout the building and not to guarantee a good geometry for positioning. For further tests, which are currently carried out, more than three Raspberry Pi units are used for a better consideration of an optimal geometry of reference stations distribution.

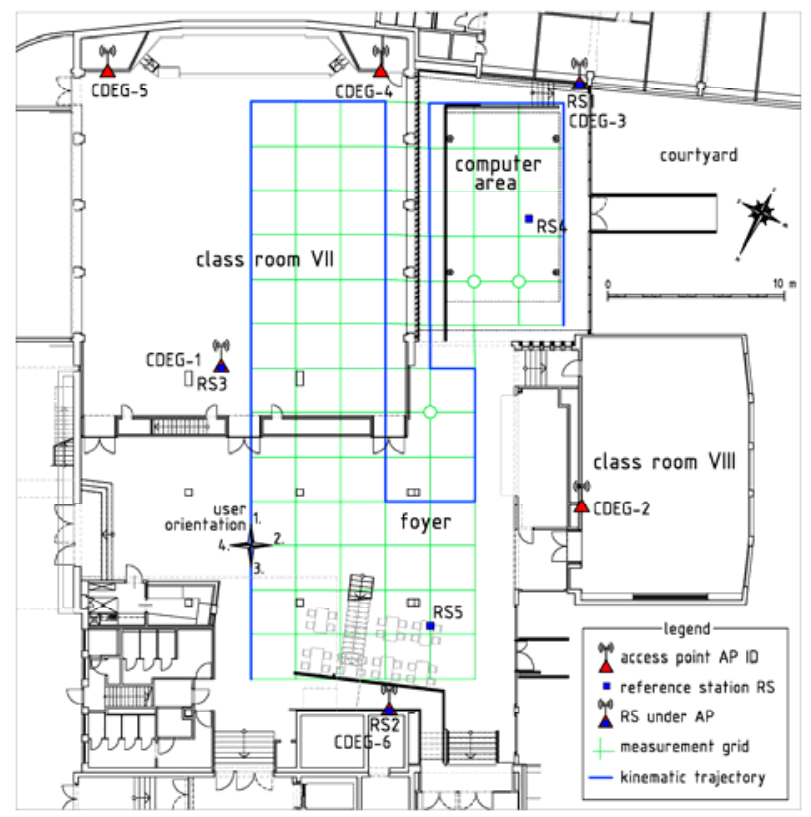

Figure 3. Map of the experimental test site

\subsection{RSS to Range Relationship}

Figure 4 shows the relationship between the range and the measured RSS on a straight line between the two APs CDEG-3 and CDEG-6 which lie $40 \mathrm{~m}$ apart. RSS measurements were performed at every $1 \mathrm{~m}$. The circles indicate the measurements from AP CDEG-3 to CDEG-6 and the triangles from AP CDEG-6 back to CDEG-3. On each point five scans in four orientations were performed, i.e., at $0^{\circ}, 90^{\circ}, 180^{\circ}$ and $270^{\circ}$, oriented in relation to the baseline between both AP's. They are indicated with different colours. The vertical error bars show the standard deviation of one sigma. As can be seen the relationship between the distance and the RSS is well described by a logarithmic function with the coefficients $a$ and $b$. When looking at the four different orientations it can be seen that the RSS is 5 to $10 \mathrm{dBm}$ lower in the case if the user is located between the AP and the mobile device, i.e., at $180^{\circ}$ orientation (blue line). The main reason for this is that the Wi-Fi signal is shielded by the human body. As already mentioned in section 2.3, Wi-Fi signals are significantly attenuated by water in the body of the smartphone user. The other orientations correspond quite well. The red dashed line is an averaged logarithmic function of these three orientations.



Figure 4. Logarithmic relationship of the RSS to the range $d$ along a straight line between two APs for one smartphone

\subsection{Static Positioning Results}

Figure 5 shows the deviations of the estimated positions from ground truth of the logarithmic one-slope model and the differential approach. The isolines correspond to the average deviations of all four orientations. For clarity, the deviations are scaled with a factor of 0.1 . The grey circles indicate that this position could not be determined and the size of the circle corresponds to the absolute number where no position estimate is obtained. For the one-slope model significant deviations of the position estimates are recognizable in the lower area in Figure 5 as objects in the foyer have a significant influence on the Wi-Fi signals and therefore also for positioning using trilateration. At some grid points in the class room VII good results with deviations of $3 \mathrm{~m}$ in maximum are achieved. With an additional use of the reference station observations an improvement of $7.5 \%$ is achieved over the whole test site. Thereby the best results are obtained in the central area of all three reference stations. Outside this triangle the errors grow due to the extrapolation of the corrections. This is expected, as differential positioning is only suitable in the area surrounded by the reference stations.
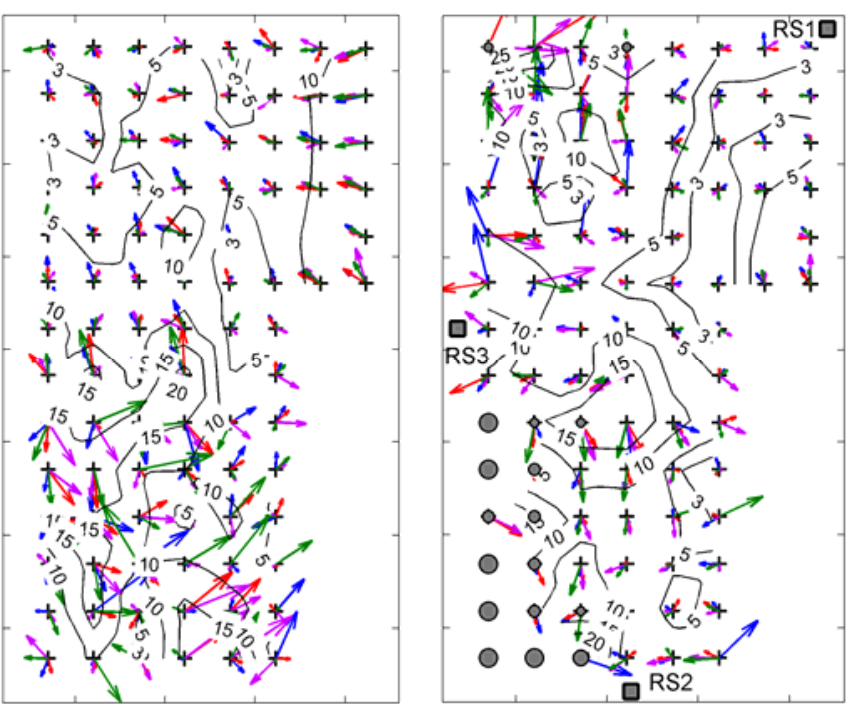

$\longrightarrow$ orientation $1 \longrightarrow$ orientation $2 \longrightarrow$ orientation $3 \longrightarrow$ orientation 4

Figure 5. Deviations of the position estimates using the logarithmic one-slope (left) and the differential approach (right) in dependence of the user's orientations for static positioning 


\subsection{Kinematic Positioning Results}

In the kinematic tests the smartphone user walked along the trajectory which is shown in blue in the map of the test site in Figure 3. As only one epoch for each location of the user is available, kinematic positioning is obviously more challenging. A comparison of the positioning result of the two methods along the trajectory is shown in Figure 6. The deviations are divided in five different classes which are indicated with different colours. As can be seen the best results are again obtained in the central area of the test site and in the computer area. In general, the resulting positioning errors are in the same order of magnitude as for static positioning. Thus, the concept of differential positioning is applicable also for kinematic positioning of a walking user.
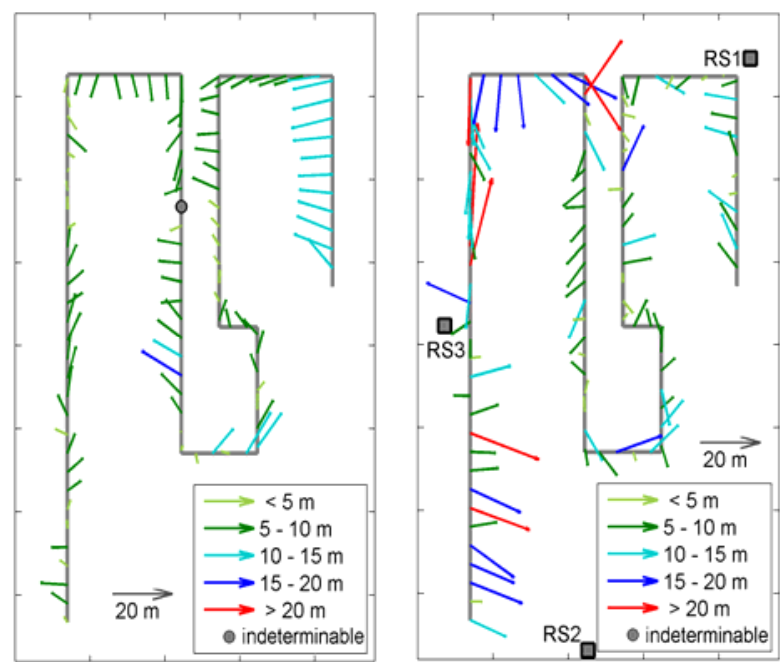

Figure 6. Deviations of the position estimates using the logarithmic one-slope (left) and the differential approach (right) for kinematic positioning

\subsection{Smartphone Comparison}
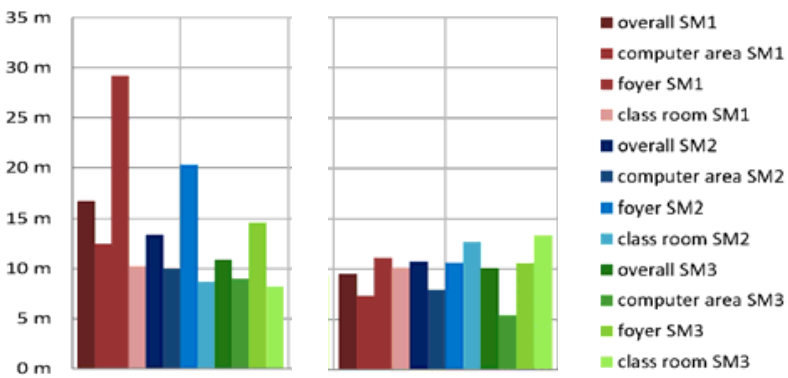

Figure 7. Comparison of the maximum deviations of the position estimates using the one-slope (left) and the differential approach (right) for three different smartphones SM1 to SM3 for static positioning

As mobile clients three different smartphones were tested (SM1, SM2 and SM3). Figures 7 shows a comparison of the maximum deviations of the position estimates using the oneslope and the differential approach for static positioning. The deviations are shown as bar graph separated again in three different areas of the test site, i.e., the area with desktop computers, the foyer and the class room. They are illustrated with different colours and colour shades. In addition, the overall deviations in the whole area of the test site are also shown. Significant differences for the deviations in the different areas can be seen whereby in the foyer the worst results are obtained.
If one looks at smartphone SM1 it can be seen that the deviations are significantly reduced if differential corrections are applied.

The results demonstrate that the DWi-Fi solution outperforms trilateration approaches where the ranges between the APs and the user are derived with simple logarithmic path loss models. The deduction of radio maps from the measured RSS values in the test site and their application for Wi-Fi positioning in a dynamically changing environment is investigated in the following sections 4 and 5 respectively.

\section{DEDUCTION OF DYNAMIC RADIO MAPS}
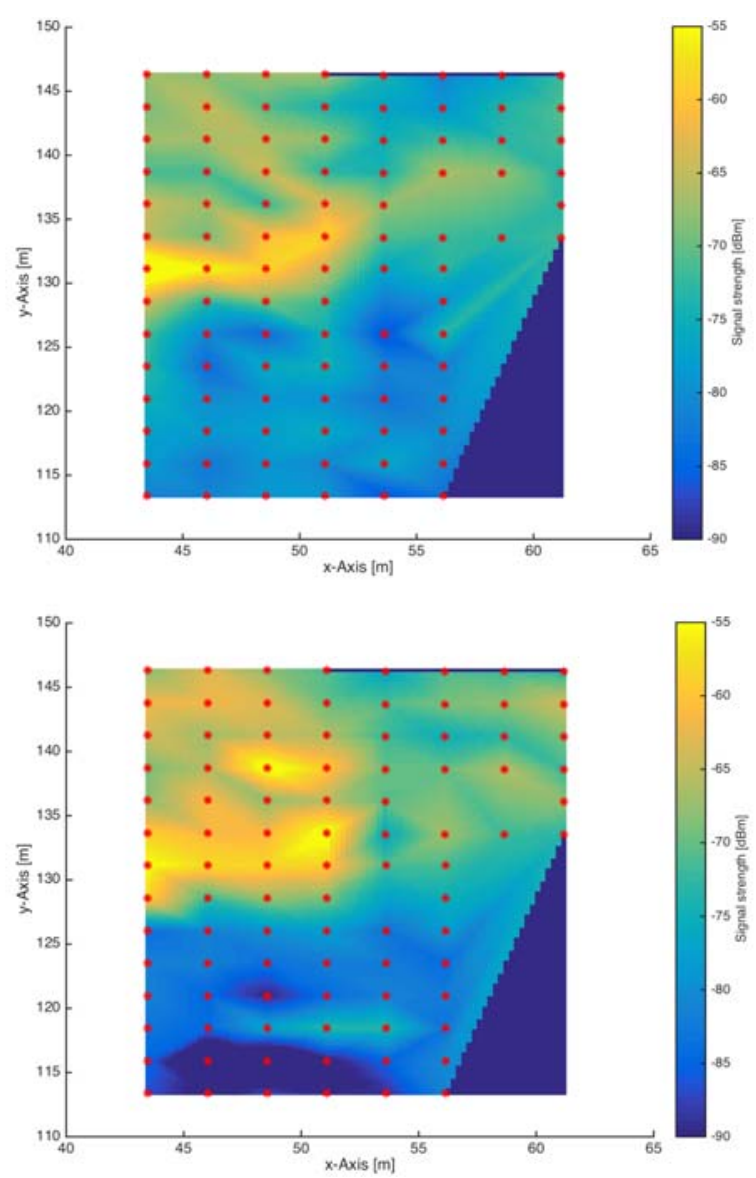

Figure 8. Example of radio maps of AP CDEG-1 in the test site for two different epochs for smartphone SM1

Radio maps of RSS distribution for the whole test area in the study are derived to be able to deduce the FKPs. For that purpose, at least three reference stations are needed. A high density for reference points has to been chosen in the tests to be able to determine the interpolation of the differential corrections at different reference grid distances. Temporal variations of the RSS are considered as measurements were performed over 24 hours. Then it is possible to analyze different time epochs with different conditions, e.g. different number of people in the building when measuring during the day and at night. As an example, Figure 8 shows the radio maps of two different time epochs of one smartphone for AP CDEG1 which is located in the class room (see Figure 3 for the location on the map in the test site). One can clearly see that regions of higher RSS are found in a rectangle which spatially matches with the class room. The RSS values are much lower behind the walls in the foyer than in the room. Apparently, the class room's walls damp a high percentage of the signal. Thus, 
a strategy for the derivation of real-time corrections for the RSS fingerprints measured at the mobile client is developed. Using this approach a significant improvement of the positioning accuracies for the mobile client is achieved. In the following, this strategy is briefly elaborated.

Using dynamically changing radio maps which are continuously updated from the observations of the network of reference stations a correction of the user's RSS observations can be estimated in real-time. The flowchart of the strategy to derive the maps in real time from previous RSS measurements is depicted in Figure 9. In step one, an average vector $\hat{\vec{r}}$ containing the RSS values derived from long-time measurements over a certain time period (e.g. one day) to the $n$ 'visible' APs is calculated to get a numerical value which describes the fluctuation of the Wi-Fi signals. This vector is representative for the average behaviour of the APs RSS. In the following step, the mean RSS vector $\vec{r}_{\overrightarrow{l t}}(t)$ for the current time epoch $t$ is calculated. Then a RSS correction vector $\vec{r}_{\text {corr }}(t)$ is derivable. Finally, the improved RSS fingerprint vector $\vec{r}_{i m p}(t)$ for the particular location of the user can be estimated.

\begin{tabular}{|c|}
\hline $\begin{array}{c}\text { Average of long-time measurements of one day } \\
\hat{\vec{r}}=\left[\widehat{R S S}^{A P_{1}}, \widehat{R S S}^{A P_{2}}, \ldots, \widehat{R S S}^{A P_{k}}\right]\end{array}$ \\
\begin{tabular}{|c|}
\hline Calculation of mean RSS \\
$\vec{r}_{l t}(t)$ for a certain epoch $t$ \\
\hline Calculation of RSS corrections for a certain epoch $t$ \\
$\vec{r}_{\text {corr }}(t)=\vec{r}_{\overline{l t}}(t)-\hat{\vec{r}}$ \\
\hline \\
\hline \\
\hline Improved RSS fingerprint \\
$\vec{r}_{\text {imp }}(t)=\vec{r}(t)+\vec{r}_{\text {corr }}(t)$ \\
\hline
\end{tabular}
\end{tabular}

Figure 9. Flowchart for the derivation of RSS corrections for the current users' location from long-time RSS measurements

Currently carried out investigations concentrate on the derivation of the required density of reference points to be able to deduce the RSS radio map. Furthermore, a comparison with location fingerprinting is performed which is the topic of the following section.

\section{FUSION CONCEPT OF FINGERPRINTING WITH TRILATERATION}

As aforementioned, a common challenge for all RSS based positioning methods are the large temporal and spatial variations of the radio channel. If dynamically updated radio maps of the area of interest are derived (as describe in the previous section and summarized in the flowchart in Figure 9) then it is possible to reduce the signal fluctuations and noise in real-time. In location fingerprinting radio maps of RSS distribution at a certain time epoch are obtained in the training phase. This is very labour consuming as a large number of reference RSS measurements need to be carried out on known points usually distributed in a rectangular grid in the area of interest. Furthermore, the training measurements needs to be repeated if the environment changes. These radio maps cannot account for short-time variations of the signal. Therefore in the new concept the radio maps are always determined and updated in real-time from the reference station measurements. This approach can be seen as fusion of location fingerprinting and trilateration as the currently determined radio maps are used to derive the ranges to the access points and reference stations. Using the derived ranges then the user's location is obtained by trilateration. A prerequisite is only that the location of the access points and reference stations need to be known. They have to be surveyed only once. As already described in section 2.4 Raspberry Pi units serve at the same time as access points and reference stations scanning and emitting Wi-Fi signals. This is a very low-cost solution.

The two radio maps in Figure 8 have been derived at two different epochs with a time interval of one hour between them. As can be seen the RSS distribution has changed and the signal strengths are significantly higher in the class room in the surrounding of AP CDEG-1 in the second epoch. Furthermore, the RSS values in the radio maps depend on the hardware of the mobile device. Figure 10 shows the radio map of a second smartphone SM2. The RSS are not that distinctive thus as with smartphone SM1. They are much smaller outside the class room in the foyer. This dependence on the used mobile device can be reduced if the radio maps derived from the Raspberry Pi units are used. A requirement is that the device must be calibrated and the relationship of the RSS scans between the mobile devices and Raspberry Pi units at the reference stations must be empirically defined. Figure 11 shows a first and second degree polynomial approximation for such a relationship for smartphone SM1. The horizontal axis describes the RSS of the Raspberry Pi relatively in the unit [\%] and the vertical the RSS of the phone in $[\mathrm{dBm}]$. It can be seen that with the quadratic approximation a slightly better result for the RSS relationship is obtained. It can therefore be recommend to use a second degree polynomial function in the calibration.

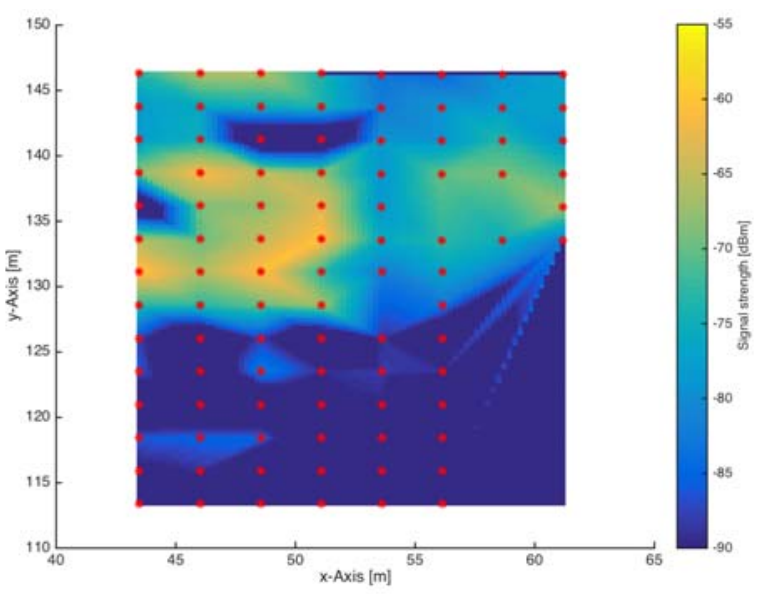

Figure 10. Radio map of AP CDEG-1 for smartphone SM2 at epoch 1

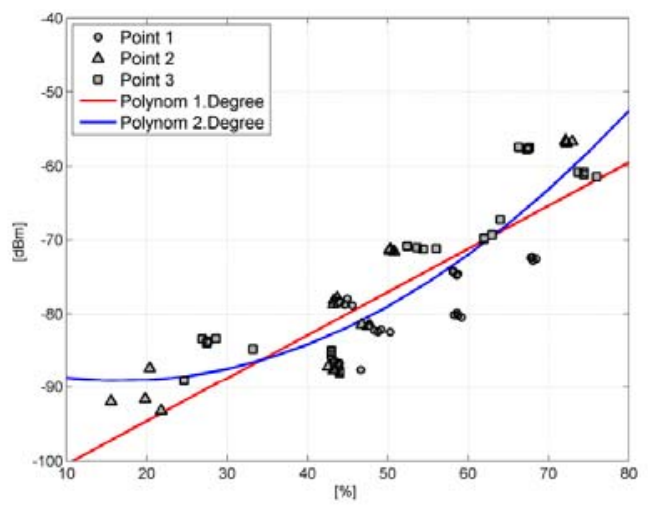

Figure 11. Polynomial approximation of RSS relationship between a Raspberry Pi unit in [\%] and smartphone SM1 in $[\mathrm{dBm}]$ 


\section{CONCLUSIONS AND OUTLOOK}

To improve the performance of Wi-Fi trilateration techniques a novel approach called Differential Wi-Fi (DWi-Fi) is derived from the well-known DGPS concept. In this approach the mobile client measures the RSS to all visible APs in the surrounding environment. Reference stations realised by Raspberry Pi units are deployed in the area of interest which measure also the RSS to all APs at the same time epoch. The major advantage of DWi-Fi is then that the RSS to range conversion is also based on areal correction parameters Flächenkorrekturparameter FKP and not only on standard theoretical path loss models. From the first test results presented in this contribution it could be proven that the novel DWi-Fi approach achieves a higher performance in terms of positioning accuracy and practicability as common logarithmic path loss models for RSS to range conversion.

The advantages of trilateration and location fingerprinting are identified and a combination is proposed. This concept differs from location fingerprinting in the fact that not at certain time intervals RSS measurements are carried out in a training phase but the radio maps are continuously updated. Due to the continuous RSS measurements at the reference stations dynamically changing radio maps are provided. They are then used to derive FKPs with an areal modeling in the network of reference stations. The user applies these FKPs which yields to an increase of positioning accuracies. In the conducted tests presented in this paper only three reference stations were used. Currently further testing with a higher number of reference stations is carried out.

Although the presented tests have been performed only indoors DWI-Fi trilateration is applicable in all environments, such as outdoors and in transition between in- to outdoors and vice versa. A seamless transition between different environments is achievable. Several current works concentrate on these developments. Thus, many different applications are feasible.

\section{REFERENCES}

Bahl, P., Padmanabhan, V. N., 2000. RADAR: An In-building RF-based User Location and Tracking System, INFOCOM 2000, In 19th Annual Joint Conference of the IEEE Computer and Communications Societies, Tel-Aviv, Vol. 2, pp. 775-784.

Chang, N., Rashidzadeh, R., Ahmadi, M., 2010. Robust Indoor Positioning Using Differential Wi-Fi Access Points. IEEE Transactions on Consumer Electronics, 56:3, pp. 1860-1867.

Chen, R., Pei, L., Liu, J., Leppäkoski, H., 2012. WLAN and Bluetooth Positioning in Smart Phones. In Chen, R. (Ed.): Ubiquitous Positioning and Mobile Location-Based Services in Smart Phones. Hershey PA, USA: IGI Global, pp. 44-68.

Gordon, N.J., Salmond, D.J., Smith, A.F.M., 1993. Novel Approach to Nonlinear/non-Gaussian Bayesian State Estimation. In IEE Proceedings F. Radar and Signal Processing, 140, pp. 107-113.

Honkavirta, V., 2008. Location Fingerprinting Methods in Wireless Local Area Networks, Master of Science Thesis, Tampere University of Technology, Finland.
Hu B., 2013. Wi-Fi Based Indoor Positioning System Using Smartphones. Master Thesis, RMIT University, Melbourne, Australia, 77 pgs.

Kotanen, A., Hännikäinen, M., Leppäkoski, H., Hämälainen, T. D., 2003. Positioning with IEEE $802.11 \mathrm{~b}$ Wireless LAN. In Proceedings of 14th International Symposium on Personal, Indoor and Mobile Radio Communications PIMRC, Beijing, China, pp. 2218-2222.

Koch, K. R., 2000. Introduction into Bayes-Statistics. Berlin Heidelberg: Springer.

Li, B., Rizos, C., 2014. Editorial: Special Issue International Conference on Indoor Positioning and Navigation 2012, Part 2, Journal of Location Based Services, 8:1: 1-2.

Liu, H., Darabi, H., Banerjee, P., Liu, J., 2007. Survey of Wireless Indoor Positioning Techniques and Systems, IEEE Transactions on Systems, Man, and Cybernetics, Part C: Applications and Reviews, 37:6: 1067-1080.

Mautz, R., 2012. Indoor Positioning Technologies, Swiss Geodetic Commission, Geodetic-Geophysical Reports of Switzerland, 86, 134 pgs.

Retscher, G., 2016. Indoor Navigation. In Grafarend, E.W. (Ed.), Encyclopedia of Geodesy. Earth Sciences Series: Springer International Publishing Switzerland, 7 pgs.

Retscher, G., Tatschl, T., 2016. Indoor Positioning Using Wi-Fi Lateration - Comparison of Two Common Range Conversion Models with Two Novel Differential Approaches. IEEE Xplore, 2016 4th International Conference on Ubiquitous Positioning, Indoor Navigation and Location Based Services (UPINLBS), Shanghai, China, 10 pgs.

Retscher, G., Zhu, M, Zhang, K., 2012. RFID Positioning. In Chen R. (ed.): Ubiquitous Positioning and Mobile LocationBased Services in Smart Phones. Hershey PA, USA: IGI Global, pp. 69-95.

Roos, T., Myllymäki, P., Tirri, H., Misikangas, P., Sievänen, J., 2002. A Probabilistic Approach to WLAN User Location Estimation. International Journal of Wireless Information Networks, 9, pp. 155-164.

Schuh, W.-D., 1984. Rasche und einfache automatische Fehlererkennung bei großen Datenmengen. Österreichische Zeitschrift für Vermessungswesen, 72:4, pp. 137-147 (in German).

Stojanović, D., Stojanović, N., 2014. Indoor Localization and Tracking: Methods, Technologies and Research Challenges, Facta Universitatis, Series: Automatic Control and Robotics, 13:1: $57-72$

Wang, N., Zhang, N., Wang, M., 2006. Wireless Sensors in Agriculture and Food Industry-Recent Development and Future Perspective. Computers and Electronics in Agriculture, 50, pp. 1-14. 